\title{
Test characteristics of quick brain MRI for shunt evaluation in children: an alternative modality to avoid radiation
}

\author{
Esther L. Yue, MD, MCR, ${ }^{1,2}$ Garth D. Meckler, MD, MSHS, MSSH, ${ }^{6}$ Ross J. Fleischman, MD, MCR, ${ }^{7}$ \\ Nathan R. Selden, MD, PhD, ${ }^{3}$ Dianna M. E. Bardo, MD, ${ }^{4}$ Amity K. Chu O'Connor, MD, ${ }^{2}$ \\ Eugene T. Vu, BS, ${ }^{2}$ Rongwei Fu, PhD, ${ }^{5}$ and David M. Spiro, MD, MPH ${ }^{1,2}$
}

\begin{abstract}
1Division of Pediatric Emergency Medicine, ${ }^{2}$ Department of Emergency Medicine, and ${ }^{3}$ Department of Neurological Surgery, Doernbecher Children's Hospital; ${ }^{4}$ Section of Pediatric Neuroradiology, Department of Diagnostic Radiology, and ${ }^{5}$ Department of Public Health and Preventive Medicine, Center for Policy and Research in Emergency Medicine, Oregon Health \& Science University, Portland, Oregon; ${ }^{6}$ Division of Pediatric Emergency Medicine, Department of Pediatrics, BC Children's Hospital, University of British Columbia, Vancouver, British Columbia, Canada; and 'Department of Emergency Medicine, Harbor University of California Los Angeles Medical Center, Torrance, California
\end{abstract}

OBJECT Quick brain magnetic resonance imaging (QB-MRI) is a rapid, radiation-free technique to detect life-threatening CSF shunt malfunction. QB-MRI has not been widely studied or adopted. The primary objective of this study was to evaluate the test characteristics of QB-MRI for detecting shunt malfunction. Test characteristics of brain computed tomography (CT) and QB-MRI were then compared. Secondary objectives included comparison of time to study completion and use of sedatives for both modalities, as well as comparison of time to study completion for QB-MRI before and after implementation of a Pediatric Emergency Department (PED) shunt clinical pathway.

METHODS A retrospective chart review was performed at 2 tertiary care hospital PEDs. The authors reviewed the charts of children who underwent QB-MRI or CT for suspected shunt malfunction between July 2008 and June 2012. They also reviewed the patients' neuroradiology reports and classified ventricular size as positive (enlarged) or negative (normal, smaller, or unchanged). Shunt malfunction was defined by surgical revision within 30 days.

RESULTS Nine hundred ninety-seven PED visits (involving 724 QB-MRIs and $273 \mathrm{CTs}$ ) were included. Surgical revision was performed in 235 cases (23.6\%). For QB-MRI, sensitivity was $58.5 \%(95 \% \mathrm{Cl} 51.1 \%-65.6 \%)$ and specificity was 93.3\% (90.8\%-95.3\%). For CT, sensitivity was $53.2 \%(95 \% \mathrm{Cl} 38.1 \%-67.9 \%)$ and specificity was $95.6 \%(92 \%-97.9 \%)$. The mean time to completion of QB-MRI was 115 minutes versus 83 minutes for CT (difference 32 minutes, $95 \% \mathrm{Cl}$, 22-42 minutes, $p<0.001$ ). The mean time from presentation to completion of QB-MRI prior to application of the CSF shunt pathway was 132 minutes versus 112 minutes after application of the CSF shunt pathway (difference 20 minutes, $95 \% \mathrm{Cl} 5-35$ minutes, $p=0.01$ ). Anxiolytic medications were used in $3.7 \%$ of CT studies and $4.4 \%$ of $Q B-M R I$ studies ( $p$ $=0.74)$.

CONCLUSIONS QB-MRI and CT have similar test characteristics for detecting CSF shunt malfunction in children and similar requirements for sedation. The longer interval from order placement to imaging completion for QB-MRI is arguably justified by reduction of radiation exposure in this population subject to frequent brain imaging.

http://thejns.org/doi/abs/10.3171/2014.9.PEDS14207

KEY WORDS magnetic resonance imaging; ventriculoperitoneal shunt; computed tomography; hydrocephalus; shunt evaluation; technique

\footnotetext{
ABBREVIATIONS ED = emergency department; OHSU = Oregon Health \& Science University; PED = pediatric ED; QB-MRI = quick brain MRI; RS-MRI = rapid-sequence MRI.

SUBMITTED April 17, 2014. ACCEPTED September 15, 2014.

INCLUDE WHEN CITING Published online January 30, 2015; DOI: 10.3171/2014.9.PEDS14207.

DISCLOSURE This publication was supported by Oregon Clinical and Translational Research Institute grant number (UL1TR000128) from the National Center for Advancing Translational Sciences at the NIH. The content is solely the responsibility of the authors and does not necessarily represent the official views of the NIH. Dr. Bardo reports a consulant relationship with Philips Healthcare.
} 
A PPROXIMATELY 125,000 children in the United States have shunt-dependent hydrocephalus, and CSF shunt-related problems account for over 15,000 pediatric hospital admissions per year. ${ }^{3,16,17}$

Because CSF shunt malfunction may be life threatening, early recognition is essential. The nonspecific nature of shunt malfunction symptoms results in partial reliance upon neuroimaging. Noncontrast brain CT is currently the standard imaging modality used in the urgent evaluation of children with shunt-dependent hydrocephalus, since it is easily accessible and contributes to rapid detection of shunt malfunction. Prior studies have estimated the sensitivity of brain CT imaging for detection of shunt malfunction to be $54 \%$ to $83 \% .^{9,10,18}$ Children with shunt-dependent hydrocephalus often undergo frequent and repeated brain imaging studies. There is growing concern about both total lifetime radiation exposure and potentially greater lifetime risk of radiation-induced cancer in children. ${ }^{4-6,11,14}$

"Quick brain" MRI (QB-MRI) was introduced in 2002 as a radiation-free alternative to conventional CT imaging for CSF shunt assessment that does not require sedation. . 7,13 Ultrafast-spin echo T2-weighted MR images, which minimize motion artifact, are acquired in 3 planes in approximately 1 minute per imaging plane, reducing the need for sedation and eliminating radiation exposure. While a promising alternative to $\mathrm{CT}$, test characteristics of QB-MRI and practical considerations, such as time required and sedation use, have not been determined.

In this study, we describe the use of QB-MRI for CSF shunt evaluation in 2 pediatric emergency departments (PEDs). The primary objective was to evaluate the test characteristics of QB-MRI for detecting shunt malfunction. We also compared the sensitivity and specificity of QB-MRI and noncontrast brain CT. Secondary objectives included comparison of time to study completion between QB-MRI and CT at a single institution. We also compared time to study completion for QB-MRI before and after implementation of a PED pathway for shunt evaluation.

\section{Methods}

\section{Study Design and Setting}

After obtaining institutional review board approval from both institutions, we reviewed patient data bases in the radiology information system to identify all patients less than 18 years of age presenting to the PED at 2 tertiary care pediatric hospitals in Portland, Oregon, who underwent imaging (shunt series radiographs, brain CT, or QB-MRI) for suspected shunt malfunction between July 2008 and June 2012. These dates were chosen based on availability of electronic health record for the two facilities. Doernbecher Children's Hospital of Oregon Health \& Science University (OHSU) was Site 1 and Randall Children's Hospital of Legacy Emanuel was Site 2. Both are located in central Portland. Site 1 has employed both brain CT and QB-MRI, while Site 2 primarily uses brain CT for shunt evaluation.

\section{Selection of Participants}

Inclusion criteria for the radiology database search included: orders placed by clinicians in the PED, noncon- trast brain CT, QB-MRI, and shunt series radiographs; age $<18$ years; July 2008 to June 2012. We used the "reason for study" for each order entry of CT or QB-MRI and only reviewed charts of patients whose images were obtained for CSF shunt evaluation. If clinical indication was not documented in the order, the electronic health record was reviewed to determine whether CSF shunt malfunction was the primary reason for the imaging study. Patients without CSF shunts were excluded. We also excluded patients whose brain imaging was obtained prior to or after their PED encounter as their images were not reliably accessible and interpreted by neuroradiologists at the two institutions. Finally, we excluded patients presenting with shunt infection after recent surgery, based on their clinical history and subsequent shunt exploration procedure.

The decision to obtain brain CT versus QB-MRI was made by the clinician in the Pediatric Emergency Department. Factors contributing to this decision included the stability of the child's condition as well as the availability of the scanners and radiology technicians. At Site 1, QBMRI was initially cumbersome to complete, making brain $\mathrm{CT}$ the preferred imaging modality. Since parents may accompany their children into the MRI scanner to calm them or to immobilize their heads during imaging, all families are required to complete a detailed MRI safety screening questionnaire before proceeding to the MRI suite. Additionally, patients with a programmable CSF shunt valve require valve reprogramming after MRI.

On August 1,2009, the PED at Site 1 instituted a clinical pathway requiring that patients presenting to the ED with symptoms concerning for CSF shunt malfunction undergo a QB-MRI within 60 minutes of presentation. Furthermore, processes for MRI safety screening and reprogramming of CSF shunt valves were streamlined. The Department of Radiology and Divisions of Pediatric Neurosurgery and Pediatric Emergency Medicine developed this clinical pathway to ensure rapid identification of children with potential CSF shunt failure and evaluation with radiation exposure limited to shunt series radiographs only. Brain CTs were obtained at the discretion of the PED clinician, sometimes in conjunction with the consulting pediatric neurosurgery service. QB-MRI was not available at Site 2 until the final week of the study period, and therefore the 1 QB-MRI study completed at Site 2 during the study period was excluded from analysis.

\section{Data Collection}

Medical chart review and data extraction were performed by 4 trained medical students and research assistants using a standardized data abstraction form. Variables collected included basic demographic characteristics, clinical information related to the PED visit, measures of Emergency Department (ED) length of stay, and requirement for sedation and/or anxiolysis during the imaging study. Clinical variables recorded were the date of visit, chief complaint, presenting symptoms, and the child's past medical and surgical history related to the CSF shunt. Since vital signs were not reliably available for data extraction, triage levels assigned by the ED were used as a proxy for stability of the patient's condition at presentation to the ED. We hypothesized that children with higheracuity triage would appear more ill and therefore be more 
likely to undergo brain CT instead of QB-MRI. Time metrics included time of arrival to the examining room, time the brain imaging was ordered, time the brain image was completed, and time of patient's disposition (admission, observation stay in the PED, or discharge home). Agents and doses used for sedation and anxiolysis, if any, were recorded.

Each image was interpreted by a neuroradiologist as per standard care. For consistency and objectivity of data review, the contemporaneous attending neuroradiologist report of the QB-MRI or CT study, and its comparison with previous studies, was used, even if neurosurgical consultant review at the time differed. This approach also reduced observer bias because, unlike the neurosurgery consultants and emergency physicians, radiologists interpreted the images independent of any patient clinical interaction. Based on these radiology reports, research assistants then classified ventricular assessment into 5 categories: 1) normal ventricular size; 2) smaller ventricles than in prior imaging study; 3) unchanged ventricular size; 4) larger ventricles compared with prior examination, concerning for shunt malfunction; and 5) enlarged ventricles but no prior comparison imaging available, concerning for shunt malfunction. Categories 1-3 were classified as negative for shunt malfunction and categories 4 and 5 were classified as positive shunt malfunction. Shunt malfunction was defined by the need for neurosurgical shunt revision within 30 days of the initial brain imaging. The two study sites are the only two institutions in the state of Oregon with staff surgeons certified or eligible for certification by the American Board of Pediatric Neurological Surgery: All subjects' medical records were reviewed to determine whether CSF shunt revision occurred at either hospital within the defined period.

\section{Outcomes}

The primary outcomes were sensitivity and specificity of QB-MRI for detecting CSF shunt malfunction. We also compared the test characteristics of QB-MRI and CT.

Secondary outcomes included the time from patient arrival in the PED examining room to placement of the imaging order; the time from order placement to completion of the imaging study; and the use and dose of sedative or anxiolytic medications during imaging. Time from imaging order placement to imaging completion was calculated as the time a CT or QB-MRI was ordered to the time the imaging study was completed in radiology. We specifically documented the time when the brain images were collected rather than the time when the images were interpreted, as the latter could vary widely based on neuroradiologist availability during the PED encounter. Time to imaging study completion was calculated as the time from the patient's arrival in the PED examining room to the completion of the brain CT or QB-MRI imaging. These time intervals were compared between cases in which CTs and QB-MRIs were obtained at Site 1, as well as before and after implementation of the shunt evaluation clinical pathway for QB-MRIs at Site 1. The requirement for sedation or anxiolysis was compared between patients examined with QB-MRI or CT. Any use of these medications for antiepileptic purposes was excluded.

\section{Data Management and Statistical Analysis}

Data were entered into a secure website, Research Electronic Data Capture (REDCap) (http://www.projectredcap.org), a database supported by the Oregon Clinical \& Translational Research Institute (OCTRI) (http://www. ohsu.edu/xd/research/centers-institutes/octri/) at OHSU. Ten percent of all records at both sites were dual reviewed for accuracy and found to have $86 \%$ agreement in data entry and review of classification of radiology reports. The majority of discrepancies were errors in time stamp entries given the transition to electronic health record for one of the study sites. Any discrepancies were reconciled by the primary investigator. All protected health information was de-identified before any statistical analysis.

The study was powered to estimate the QB-MRI sensitivity with a $95 \%$ CI limit of $\pm 7 \%$ (shunt revisions $n$ $=200$, assuming the sensitivity is approximately 60\%). The number of shunt revisions based on CT was expected to be substantially lower than the number based on QBMRI because a priori it was hypothesized that fewer CTs were ordered after the CSF shunt clinical pathway was implemented at Site 1. We compared test characteristics between QB-MRI and CT for exploratory purposes, and this study was not powered to detect the differences in sensitivity and specificity between QB-MRI and CT.

Descriptive statistics were used to summarize patient characteristics. Test characteristics for brain CT and QBMRI were examined using sensitivity and specificity. Pearson's chi-square test was used to compare test characteristics, shunt failure, and use of sedating or anxiolytic medications between the brain CT and QB-MRI cohorts. Since test characteristics for brain CT between Site 1 and Site 2 cohorts were very similar, they were combined in the final analysis to increase the sample size of the CT cohort. Comparison of time to image completion between brain CT and QB-MRI and comparison of time to QBMRI completion before and after the implementation of the shunt clinical pathway at Site 1 were performed using a 2-sample t-test. All analyses were conducted using SAS 9.3 (SAS Institute Inc.) except the calculation of sensitivity and specificity, for which Stata/IC 11 (StataCorp LP) was used.

\section{Results}

\section{Patient Characteristics}

In total, 1435 charts were reviewed, with 438 excluded based on exclusion criteria, in most cases because brain imaging was completed prior to the patient's PED visit (Fig. 1). In the remaining 997 cases, 134 brain CTs were obtained at Site 2, 139 brain CTs at Site 1, and 724 QBMRIs were collected at Site 1 for shunt evaluation. The mean age of the patients was 7.1 years (range 22 days to 17.9 years), and $62.7 \%$ were boys. Patient characteristics stratified by imaging cohort are presented in Table 1;1.8\% of the CT cohort and $0.1 \%$ of the QB-MRI cohort were triaged to Level 1 (critical), while $35.5 \%$ of the CT cohort and $63 \%$ of the QB-MRI cohort were triaged to Level 2 (emergent). At Site 1, the proportion of patients with surgically confirmed shunt failure was $26.0 \%$ (188 of 724) in the QB-MRI group and 27.3\% in the CT group (38 of 139; $\mathrm{p}=0.74)$. 


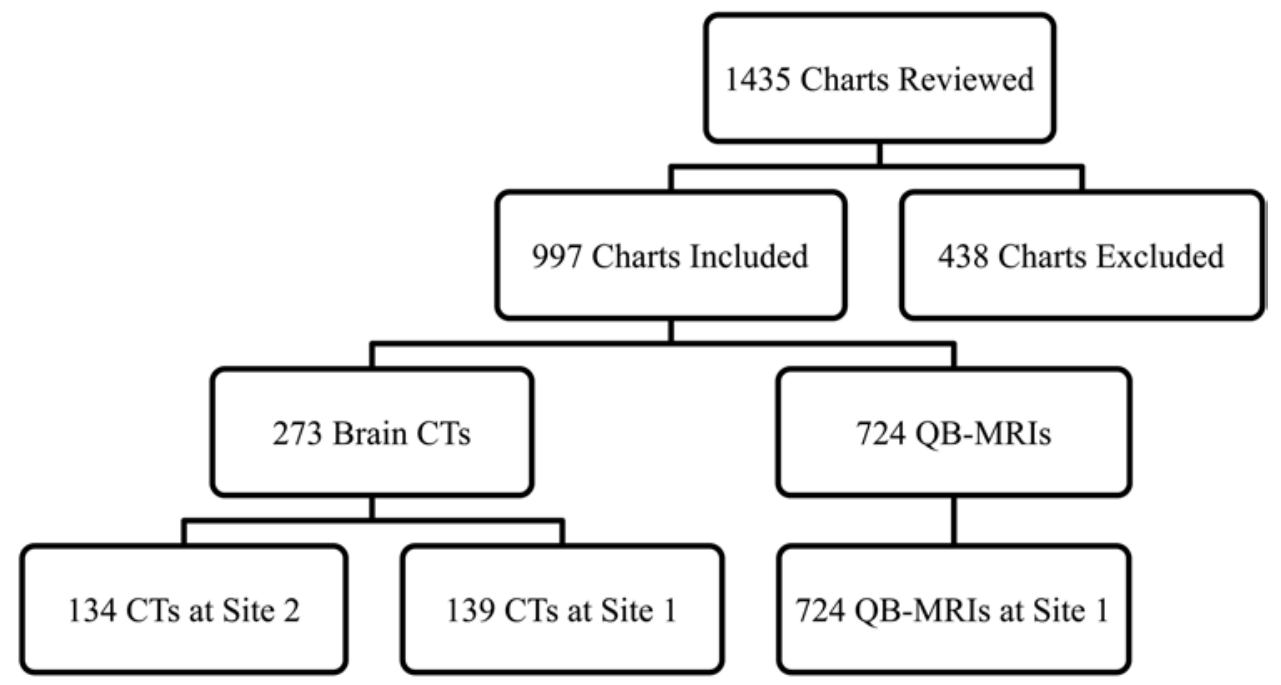

FIG. 1. Brain imaging in PEDs for shunt evaluation from July 2008 to June 2012.

\section{Test Characteristics}

There were a total of 188 shunt revisions in the QBMRI group; the sensitivity to detect a shunt failure was $58.5 \%$ (95\% CI 51.1\%-65.6\%), and the specificity was 93.3\% (95\% CI 90.8\%-95.3\%). In the CT group, there were a total of 47 shunt revisions at both sites; the sensitivity was $53.2 \%$ (95\% CI $38.1 \%-67.9 \%$ ), and specificity was 95.6\% (95\% CI 92\%-97.9\%). There was no statistically significant difference between QB-MRI and CT with respect to sensitivity $(\mathrm{p}=0.51)$ or specificity $(\mathrm{p}=0.23)$.

\section{Time to Imaging Study Completion}

Time to imaging study completion was only measured at Site 1, as confounding factors made comparison between the two sites difficult. During the study period, 139 CT studies and 724 QB-MRI studies were completed at Site 1. Although it took longer for PED clinicians at Site 1 to order a CT study as compared with QB-MRI (mean 51 vs 32 minutes, $p<0.001$ ), the average time from presentation to completion of CT was 83 minutes, compared with 115 minutes for QB-MRI (mean difference 32 minutes, $95 \%$ CI 22-42 minutes, $p<0.001$, Fig. 2). This difference was accounted for by a longer interval from order placement to imaging completion for QB-MRI.

For the comparison of time to imaging before and after implementation of the CSF shunt evaluation clinical pathway at Site 1, 95 QB-MRI studies were completed before the implementation of the CSF shunt evaluation pathway, with a mean time of 132 minutes, and 629 QB-MRI studies were completed afterward, with a mean time of 112 minutes (20 minute reduction post-implementation, 95\% CI 5-35 minutes, $\mathrm{p}=0.01$ ). While time from QB-MRI order placement to imaging study completion remained essentially the same (81 vs 84 minutes, $p=0.61$ ), there was a significant reduction in time from patient presentation to the QB-MRI order placement from 52 minutes before compared with 28 minutes after establishment of the shunt pathway ( $<<0.001$, Fig. 3). The number of CT studies completed quarterly declined while the number of
QB-MRI studies completed increased after implementation of the shunt clinical pathway (Fig. 4).

\section{Use of Anxiolysis or Sedation}

There was no statistically significant difference in the use of medications for sedation or anxiolysis for CT compared with QB-MRI (3.7\% of CTs and 4.4\% of QB-MRIs, $\mathrm{p}=0.74$, Table 2). The most common medication administered was either midazolam (administered orally or intranasally).

\section{Discussion}

Our results suggest that QB-MRI and CT have similar test characteristics for detecting CSF shunt malfunction in children presenting to the PED. Our findings regarding the test characteristics for CT are similar to those described previously. Mater et al. ${ }^{10}$ and Lehnert et al. ${ }^{9}$ described sensitivity and specificity of CT as $61 \%$ and $83 \%$, and $54 \%$ and $90 \%$, respectively. In our study, the sensitivity and specificity of QB-MRI were not significantly different from that of CT for shunt evaluation. In earlier studies, Iskandar et al.,7 Ashley et al., ${ }^{2}$ O'Neill et al., ${ }^{13}$ Rozovsky et al., ${ }^{15}$ and Niderhauser et al. ${ }^{12}$ reported on image quality, catheter visualization, and image acquisition time for QBMRI for shunt-dependent hydrocephalus (also referred to as rapid-sequence MRI [RS-MRI] or rapid brain MRI). The acquisition time and procedure time for QB-MRI reported by prior studies are similar to ours. Our examination consists of 4 sequences: a scout image, which requires approximately 30 seconds, followed by an axial, sagittal, and coronal sequence, which require approximately 45 seconds each. The acquisition time is therefore $2.75 \mathrm{~min}$ utes, but with a few seconds between each scan, the total imaging procedure time is approximately $3-5$ minutes long.

The charges for QB-MRI and CT at Site 1 are similar to the prices published by O'Neill et al. ${ }^{13}$ The technical and professional fees for their RS-MRI were $\$ 1800$ and $\$ 170$, respectively, and the corresponding fees for CT were 
TABLE 1. Patient characteristics in 997 cases

\begin{tabular}{|c|c|c|}
\hline \multirow[b]{2}{*}{ Characteristic } & \multicolumn{2}{|c|}{ Value $^{*}$} \\
\hline & $C T(n=273)$ & QB-MRI $(n=724)$ \\
\hline Age in years, mean (SD) & $7.1(4.5)$ & $7.1(5.1)$ \\
\hline \multicolumn{3}{|l|}{ Sex } \\
\hline Female & $102(37.4)$ & $270(37.3)$ \\
\hline Male & $171(62.6)$ & $454(62.7)$ \\
\hline \multicolumn{3}{|l|}{ Chief complaint } \\
\hline Headache & $69(25.3)$ & $259(35.8)$ \\
\hline Vomiting & $84(30.8)$ & $233(32.2)$ \\
\hline Fever & $48(17.6)$ & $80(11.0)$ \\
\hline Altered mental status & $24(8.8)$ & $60(8.3)$ \\
\hline Fussiness/irritability & $9(3.3)$ & $66(9.1)$ \\
\hline Seizure & $50(18.3)$ & $57(7.9)$ \\
\hline Abdominal pain & $16(5.9)$ & $33(4.6)$ \\
\hline Shunt evaluation & $22(8.1)$ & $226(31.2)$ \\
\hline Swelling/redness over shunt & $5(1.8)$ & $52(7.2)$ \\
\hline Trauma or fall & $13(4.8)$ & $10(1.4)$ \\
\hline Difficulty breathing & $11(4.0)$ & $14(1.9)$ \\
\hline Heart rate abnormality & $1(0.4)$ & $4(0.6)$ \\
\hline Diarrhea & $3(1.1)$ & $9(1.2)$ \\
\hline Focal neurologic problem & $16(5.9)$ & $45(6.2)$ \\
\hline Fatigue & $3(1.1)$ & $25(3.5)$ \\
\hline Other & $8(2.9)$ & $44(6.1)$ \\
\hline \multicolumn{3}{|l|}{ Type of shunt } \\
\hline Ventriculoatrial & $2(0.7)$ & $16(2.2)$ \\
\hline Ventriculopleural & $7(2.6)$ & $21(2.9)$ \\
\hline Ventriculoperitoneal & $264(96.7)$ & $687(94.9)$ \\
\hline \multicolumn{3}{|l|}{ Reason for shunt } \\
\hline Hydrocephalus & $250(91.6)$ & $669(92.4)$ \\
\hline Intracranial bleed & $35(12.8)$ & $149(20.6)$ \\
\hline Myelomeningocele & 33 (12.1) & $130(18.0)$ \\
\hline Spina bifida & $17(6.2)$ & $145(20.0)$ \\
\hline Tumor resection & $14(5.1)$ & $63(8.7)$ \\
\hline \multicolumn{3}{|l|}{ Triage level } \\
\hline 1 (Critical) & $5(1.8)$ & $1(0.1)$ \\
\hline 2 (Emergent) & $97(35.5)$ & $456(63.0)$ \\
\hline 3 (Urgent) & $139(50.9)$ & $263(36.3)$ \\
\hline 4 (Non-urgent) & $24(8.8)$ & $4(0.6)$ \\
\hline \multicolumn{3}{|l|}{ Attending specialty } \\
\hline Emergency medicine & $109(39.9)$ & $370(51.1)$ \\
\hline Nurse practitioner & $8(2.9)$ & $66(9.1)$ \\
\hline $\begin{array}{l}\text { Pediatric emergency medi- } \\
\text { cine }\end{array}$ & $156(57.1)$ & $288(40.0)$ \\
\hline
\end{tabular}

QB-MRI = quick brain MRI.

* Values represent numbers of cases (\%) unless otherwise indicated.

$\$ 1200$ and \$123. Their RS-MRI was \$647 more expensive than CT per imaging session. At our institution, the technical and professional fees for QB-MRI are \$1650 and $\$ 179$, respectively, and the fees for CT are $\$ 1070$ and $\$ 102$. Our QB-MRI is $\$ 657$ more expensive than CT. The higher cost of MRI compared with CT is multifactorial and

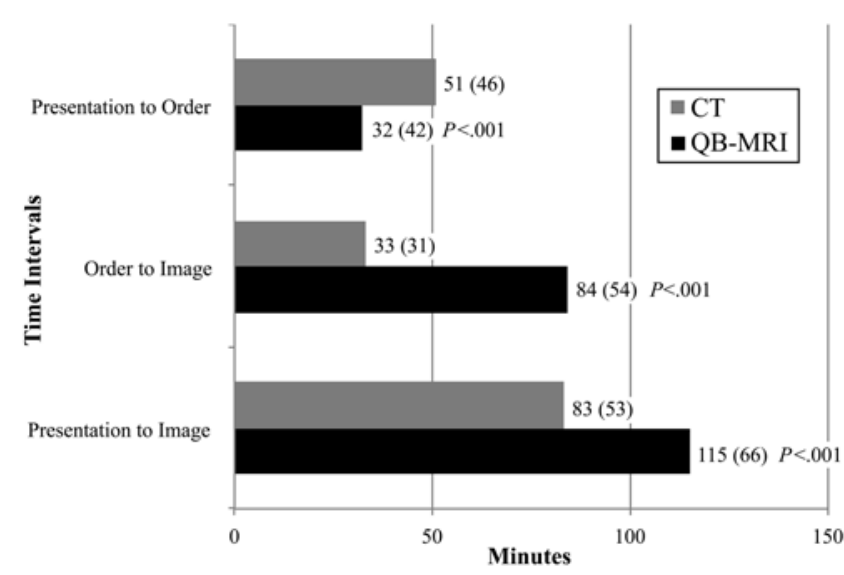

FIG. 2. Time metrics of Site 1 CT vs QB-MRI (mean [SD]). Time from presentation to order placement: mean difference 19 minutes $(95 \% \mathrm{Cl}$ 11-27 minutes, $p<0.001)$. Time from order placement to image completion: mean difference 51 minutes (95\% Cl 45-58 minutes, $p<0.001)$. Time from presentation to image completion: mean difference $32 \mathrm{~min}$ utes $(95 \% \mathrm{Cl} 22-42$ minutes, $p<0.001)$

reflects higher technology and personnel costs and more image planes for acquisition and interpretation in MRI compared with CT.

Considering the potential radiation risks associated with the use of $\mathrm{CT}, 11,14$ our findings have important implications for the care of children with suspected shunt malfunction. There is wide variation in the number of brain imaging studies a child with shunt-dependent hydrocephalus will undergo in his or her lifetime, and it is therefore difficult to predict the exact amount of effective radiation dose saved per patient. Koral et al. estimate the annual frequency of surveillance neuroimaging in this patient population to be approximately 2 CTs per year throughout a lifetime. ${ }^{8}$ An individual patient in our study underwent 25 brain imaging studies during the 4-year study period, and 21 of these 25 imaging studies were QB-MRI studies.

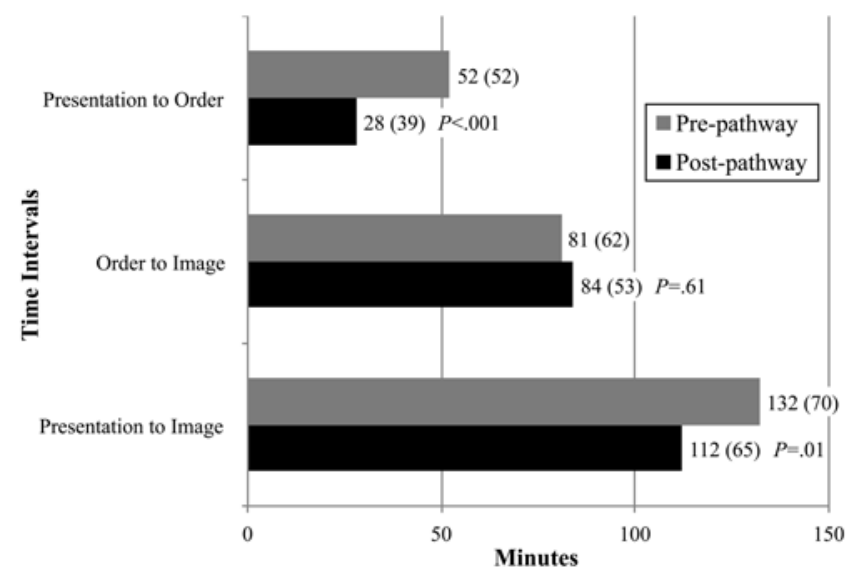

FIG. 3. Time metrics of Site $1 \mathrm{QB}-\mathrm{MRI}$ before and after implementation of the shunt clinical pathway (mean [SD]). Time from presentation to order placement: mean difference 24 minutes $(95 \% \mathrm{Cl} 12-34$ minutes, $p$ $<0.001)$. Time from order placement to image completion: mean difference 3 minutes $(p=0.61)$. Time from presentation to image completion: mean difference 20 minutes $(95 \% \mathrm{Cl} 5-35$ minutes, $p=0.01)$. 


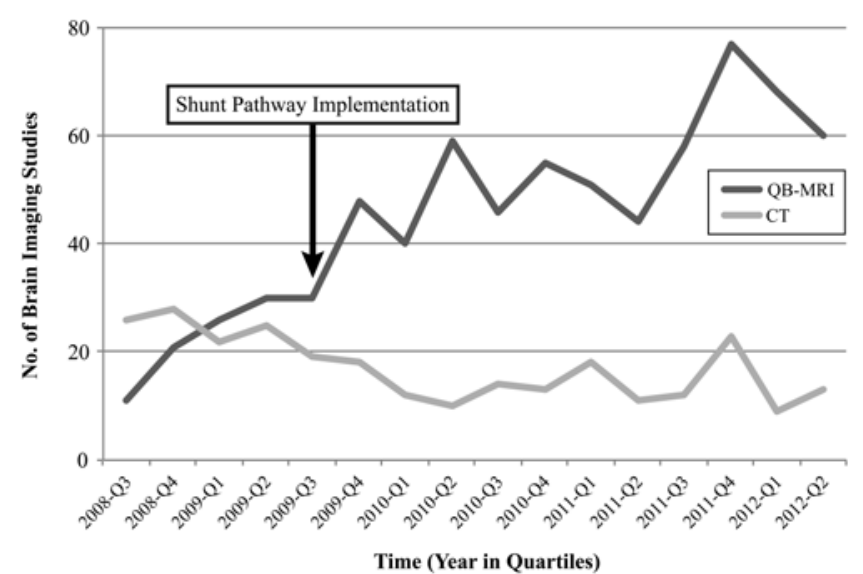

FIG. 4. Use of CT and QB-MRI over the 4-year study period at the Site 1 PED.

With an estimate of $2-5 \mathrm{mSv}$ administered per brain CT, this child was spared $42-105 \mathrm{mSv}$ of effective radiation dose during our study period alone through the use of QBMRI. In its recent Position Statement on Radiation Risks of Medical Imaging Procedures, the American Association of Physicists in Medicine (AAPM) states that "risks of medical imaging at effective doses below $50 \mathrm{mSv}$ for single procedures or $100 \mathrm{mSv}$ for multiple procedures over short time periods are too low to be detectable and may be nonexistent." Although controversial, the AAPM statement highlights that there remains concern for patients receiving cumulative effective doses above those stated. Clearly, patients with CSF shunts who undergo repetitive CT imaging studies, such as the patients in our study, often fall within the at-risk population.

A limitation of traditional MRI is the need for procedural sedation or anxiolysis in younger children; QB-MRI can be acquired quickly and overcomes this limitation. We found a similar rate of use of sedative or anxiolytic medications for the two imaging modalities, suggesting that QB-MRI does not require more sedation than CT. Additional methods to avoid sedation at both study sites include swaddling and bottle-feeding for young infants and distraction by parents and child life specialists for toddlers and older children. Niederhauser et al. reviewed rapid brain MRI examinations in non-sedated children and

TABLE 2. Use of anxiolytic or sedative medication for CT vs QB-MRI

\begin{tabular}{lcc}
\hline & \multicolumn{2}{c}{ No. of Cases (\%) } \\
\cline { 2 - 3 } Anxiolytic or Sedative & $\mathrm{CT}(\mathrm{n}=273)$ & QB-MRI $(\mathrm{n}=724)$ \\
\hline Intranasal midazolam & $0(0)$ & $9(1.2)$ \\
\hline Intranasal fentanyl & $0(0)$ & $0(0)$ \\
\hline Intravenous lorazepam & $1(0.4)$ & $2(0.3)$ \\
\hline Intravenous midazolam & $4(1.5)$ & $6(0.8)$ \\
\hline Intravenous ketamine & $1(0.4)$ & $0(0)$ \\
\hline Intravenous propofol & $1(0.4)$ & $2(0.3)$ \\
\hline Oral lorazepam & $0(0)$ & $0(0)$ \\
\hline Oral midazolam & $3(1.1)$ & $12(1.7)$ \\
\hline Total & $10(3.7)$ & $32(4.4)$ \\
\hline
\end{tabular}

found adequate diagnostic visualization of ventricular size in nearly 400 examinations. ${ }^{12}$

At Site 1, the estimated imaging time for noncontrast brain CT is 5 minutes with an actual examination time of 5-10 seconds versus the estimated imaging time of 3-5 minutes for QB-MRI with an examination time of $45 \mathrm{sec}-$ onds per plane, using axial, sagittal, and coronal imaging planes. The vast majority of patients did not require moderate or deep sedation for either modality. Only 4 patients required procedural sedation for either QB-MRI or brain $\mathrm{CT}$. We found no significant difference in the number of shunt failures in the CT or QB-MRI cohorts.

On average, brain CTs were completed 32 minutes more quickly than QB-MRIs from the time a patient arrived in the PED room to completion of imaging. QB-MRI studies were ordered on average 19 minutes faster than CT studies, but patients had to wait on average 51 minutes longer to access the MRI scanner as compared with the CT scanner. These time differences were all statistically significant ( $p<0.001)$. As use of QB-MRI for CSF shunt evaluation became standard at Site 1, children were selected for CT imaging only if they could not tolerate QB-MRI or if QB-MRI was contraindicated. Contraindications for QB-MRI include implanted objects such as pacemakers, implanted cardioverter defibrillators, or cochlear implants. We postulate that the delay in ordering CTs may be due to discussion between the PED clinician and neurosurgery consultant regarding the actual need for $\mathrm{CT}$ in an effort to decrease radiation exposure in these remaining children.

As the actual examination times for brain CT and QBMRI are comparable, we hypothesize that the reason for the longer time interval from order placement to examination completion could be the relative proximity of CT scanners to the PED, compared with MRI scanners, which are located several floors away. Further, as traditional MRI studies require longer imaging times and other patients are scheduled on a routine basis throughout the day, the availability of MRI scanners for QB-MRI patients may be temporarily limited in some instances. Wait times for CT scanners are typically shorter, at 5-15 minutes, compared with wait times for MRI scanners, ranging from 30 to 120 minutes.

Lastly, based on this study, the implementation of the Pediatric Emergency Department shunt evaluation clinical pathway at Site 1 decreased the time to brain imaging completion for QB-MRI by 20 minutes, with most of that time difference accounted for by faster ED order times.

\section{Limitations}

The primary limitation of this study is its retrospective methodology, which may lead to incorrect classifications, incomplete documentation, and missing data. Additionally, since our subject list was created through the radiology database at our institutions, patients with brain imaging completed at referring facilities were not included. Lack of prospective follow-up may have also allowed children to be misclassified, but this is unlikely, as the region's only pediatric neurosurgical services exist in the two study centers.

There are limitations of QB-MRI such as the decreased ability to see blood products and air, and sometimes it is difficult to see ventricular catheter position, especially 
with small ventricles. However QB-MRI has typically been recommended only to investigate possible shunt failure, ventriculomegaly, hydrocephalus, and fluid collections such as arachnoid cysts.

In practice, the diagnosis of CSF shunt malfunction is made on clinical grounds, often relying on a combination of symptoms, physical examination findings, and neuroimaging findings. Shunt malfunction was defined in our study as operative shunt revision within 30 days. Since the neurosurgeons were not blinded to the results of the imaging studies, there was potential for bias in determining the diagnosis of shunt malfunction, although this would have been similar for both the CT and QB-MRI group.

\section{Conclusions}

In summary, while larger prospective studies are needed to validate our findings, QB-MRI should be considered in the urgent evaluation of CSF shunts to avoid unnecessary radiation exposure. CT and QB-MRI have similar test characteristics in detecting shunt malfunction and a similar requirement for sedation and/or anxiolysis. Institution of a clinical guideline reduced the time to clinician order placement for QB-MRI but did not alter the time to complete the study once an order was placed. CT is completed more quickly than QB-MRI, a difference primarily accounted for by a longer interval from order placement to image completion for QB-MRI. QB-MRI, however, avoids the risks of ionizing radiation exposure in this population of children subject to frequent brain imaging.

\section{References}

1. American Association of Physicists in Medicine: AAPM Position Statement on Radiation Risks From Medical Imaging Procedures. (https://www.aapm.org/org/policies/details. asp?id=318\&type $=P P \&$ current=true) [Accessed December 2, 2014]

2. Ashley WW Jr, McKinstry RC, Leonard JR, Smyth MD, Lee BC, Park TS: Use of rapid-sequence magnetic resonance imaging for evaluation of hydrocephalus in children. J Neurosurg 103 (2 Suppl): 124-130, 2005

3. Berry JG, Lieu TA, Forbes PW, Goldmann DA: Hospital volumes for common pediatric specialty operations. Arch Pediatr Adolesc Med 161:38-43, 2007

4. Brenner D, Elliston C, Hall E, Berdon W: Estimated risks of radiation-induced fatal cancer from pediatric CT. AJR Am J Roentgenol 176:289-296, 2001

5. Brenner DJ, Hall EJ: Computed tomography-an increasing source of radiation exposure. N Engl J Med 357:2277-2284, 2007

6. Brody AS, Frush DP, Huda W, Brent RL: Radiation risk to children from computed tomography. Pediatrics 120:677682,2007

7. Iskandar BJ, Sansone JM, Medow J, Rowley HA: The use of quick-brain magnetic resonance imaging in the evaluation of shunt-treated hydrocephalus. J Neurosurg 101 (2 Suppl):147-151, 2004

8. Koral K, Blackburn T, Bailey AA, Koral KM, Anderson J: Strengthening the argument for rapid brain MR imaging: estimation of reduction in lifetime attributable risk of developing fatal cancer in children with shunted hydrocephalus by instituting a rapid brain MR imaging protocol in lieu of head CT. AJNR Am J Neuroradiol 33:1851-1854, 2012

9. Lehnert BE, Rahbar H, Relyea-Chew A, Lewis DH, Richard- son ML, Fink JR: Detection of ventricular shunt malfunction in the ED: relative utility of radiography, CT, and nuclear imaging. Emerg Radiol 18:299-305, 2011

10. Mater A, Shroff M, Al-Farsi S, Drake J, Goldman RD: Test characteristics of neuroimaging in the emergency department evaluation of children for cerebrospinal fluid shunt malfunction. CJEM 10:131-135, 2008

11. Mathews JD, Forsythe AV, Brady Z, Butler MW, Goergen SK, Byrnes GB, et al: Cancer risk in 680,000 people exposed to computed tomography scans in childhood or adolescence: data linkage study of 11 million Australians. BMJ 346:f 2360,2013

12. Niederhauser BD, McDonald RJ, Eckel LJ, Keating GF, Broomall EM, Wetjen NM, et al: Retrospective review of rapid pediatric brain MR imaging at an academic institution including practice trends and factors affecting scan times. AJNR Am J Neuroradiol 34:1836-1840, 2013

13. O’Neill BR, Pruthi S, Bains H, Robison R, Weir K, Ojemann $\mathrm{J}$, et al: Rapid sequence magnetic resonance imaging in the assessment of children with hydrocephalus. World Neurosurg 80:e307-e312, 2013

14. Pearce MS, Salotti JA, Little MP, McHugh K, Lee C, Kim $\mathrm{KP}$, et al: Radiation exposure from CT scans in childhood and subsequent risk of leukaemia and brain tumours: a retrospective cohort study. Lancet 380:499-505, 2012

15. Rozovsky K, Ventureyra EC, Miller E: Fast-brain MRI in children is quick, without sedation, and radiation-free, but beware of limitations. J Clin Neurosci 20:400-405, 2013

16. Simon TD, Riva-Cambrin J, Srivastava R, Bratton SL, Dean JM, Kestle JR: Hospital care for children with hydrocephalus in the United States: utilization, charges, comorbidities, and deaths. J Neurosurg Pediatr 1:131-137, 2008

17. Stein SC, Guo W: The prevalence of shunt-treated hydrocephalus: a mathematical model. Surg Neurol 72:131-137, 2009

18. Zorc JJ, Krugman SD, Ogborn J, Benson J: Radiographic evaluation for suspected cerebrospinal fluid shunt obstruction. Pediatr Emerg Care 18:337-340, 2002

\section{Author Contributions}

Conception and design: Yue, Meckler, Selden, Bardo, Spiro. Acquisition of data: Yue, Chu O'Connor, Vu. Analysis and interpretation of data: Yue, Fleischman, Fu. Drafting the article: Yue. Critically revising the article: Meckler, Fleischman, Selden, Bardo, Chu O'Connor, Vu, Fu, Spiro. Reviewed submitted version of manuscript: all authors. Approved the final version of the manuscript on behalf of all authors: Yue. Statistical analysis: Fu. Study supervision: Yue, Spiro.

\section{Supplemental Information \\ Previous Presentation}

Portions of this work were presented in abstract form at the Pediatric Academic Societies' Meeting, Washington, DC, May 2013, and at the Society for Academic Emergency Medicine Annual Meeting, Atlanta, Georgia, May 2013. Portions of this work were also presented at the American Academy of Pediatrics National Conference and Exhibitions, Orlando, Florida, October 2013, and at the American Association of Neurological Surgeons Section on Pediatric Neurological Surgery Annual Scientific Meeting, Toronto, Ontario, Canada, December 2013.

\section{Correspondence}

Esther L. Yue, Department of Emergency Medicine, Division of Pediatric Emergency Medicine, Doernbecher Children's Hospital, Oregon Health \& Science University, 3181 SW Sam Jackson Park Rd., Portland, OR 97239. email: yue@ohsu.edu. 